\title{
First-Order Transition and Critical End-Point in Vortex Liquids in Layered Superconductors
}

\author{
Joonhyun YEO* \\ Department of Physics, Konkuk University, Seoul, Korea 143-701 \\ M. A. Moore \\ Department of Physics, University of Manchester, Manchester M13 9PL, United Kingdom
}

(November 12, 2018)

\begin{abstract}
We calculate various thermodynamic quantities of vortex liquids in a layered superconductor by using the nonperturbative parquet approximation method, which was previously used to study the effect of thermal fluctuations in two-dimensional vortex systems. We find there is a first-order transition between two vortex liquid phases which differ in the magnitude of their correlation lengths. As the coupling between the layers increases, the first-order transition line ends at a critical point. We discuss the possible relation between this critical end-point and the disappearance of the firstorder transition which is observed in experiments on high temperature superconductors at low magnetic fields.
\end{abstract}

\section{INTRODUCTION}

Thermal fluctuations and quenched disorder are two important factors responsible for the rich phase diagram exhibited by high temperature superconductors in a magnetic field [1]. Recent experiments [2, 3] on high temperature superconductors show a first-order transition line well below the upper critical field $H_{c 2}(T)$ in the field-temperature (H-T) phase diagram. When the strength of disorder is weak, this line is usually interpreted as a melting line of a vortex lattice into a vortex liquid. The first-order transition disappears at both high and low magnetic fields 沺曰. In the vortex lattice melting picture the loss of first-order behavior at high fields is usually attributed to the effect of disorder, which is supposed somehow to produce a multi-critical point where the first-order transition changes into a continuous one.

In a recent numerical simulation [8] of a vortex system in a layered superconductor, a first-order transition line was also obtained which disappeared at a critical end-point at low magnetic fields. According to the simulation results, in contrast to the vortex lattice melting picture, there is only one phase below and above the transition, namely the vortex liquid but with different correlation lengths on either side of the first-order transition line. However, in other simulations [9] using periodic boundary conditions within a layer, an apparent first-order transition between vortex liquid and lattice was obtained.

In this paper we apply an analytical approach to a layered superconductor in a magnetic field perpendicular to the layers in an attempt to elucidate the nature of phase transitions in the system. We use the parquet approximation [10], which has been successfully applied to the two-dimensional vortex system. It is a nonperturbative analytic method free from any finite size or boundary effects in the direction perpendicular to the magnetic field. The parquet approximation deals with the renormalized four-point function of the vortex system which is obtained by summing an infinite subset of Feynman diagrams, the so-called parquet diagrams. In two dimensions this method is able to capture the growing crystalline order developing in the vortex liquid as the temperature is lowered [10]. As explained in the discussion below, the introduction of an additional dimension to the parquet calculations imposes severe numerical difficulties because of the large number of variables specifying the renormalized vertex function. This problem has restricted us in this paper to considering a small system which consists of just four layers satisfying a periodic boundary condition along the field direction. Each layer is, however, treated as an infinite plane so we can are in effect descibing an infinite number of vortices. Despite the unphysically small size of the system we consider here, we

*E-mail : jhyeo@konkuk.ac.kr 
find that the effect of the inter-layer couplng makes a significant difference compared with the two-dimensional case, where no finite-temperature phase transition occurs in the parquet calculation. In the layered system, we find that the thermodynamic quantities describing the vortex liquid show abrupt changes when the field and the temperature are varied, and these sharp changes are interpreted as a first-order phase transition within the vortex liquid phase. We find that the first-order transition line ends at a critical end-point. The two phases we obtain above and below the transition are both liquid phases with different correlation lengths, consistent with the numerical simulation results [8].

In the next section we present our model for the vortex liquid in a layered superconductor. We then set up the parquet equations for the layered system. In the final section we present the numerical solutions of the parquet equation for the four-layer system which are interpreted in terms of a first-order transition and the termination of the first-order transition line at a critical end-point. We conclude with a discussion on the possible implication of our results for the H-T phase diagram of a layered superconductor.

\section{MODEL}

Our starting point is the Lawrence-Doniach model for a layered superconductor in a magnetic field perpendicular to the layers. With the order parameter in the $\mathrm{n}$-th layer denoted by $\psi_{n}$, the free energy is given by

$$
\begin{gathered}
F\left[\psi, \psi^{*}\right]=\sum_{n} d_{0} \int d^{2} \mathbf{r}\left[\alpha\left|\psi_{n}(\mathbf{r})\right|^{2}+\frac{\beta}{2}\left|\psi_{n}(\mathbf{r})\right|^{4}\right. \\
+\frac{1}{2 m_{a b}}\left|\left(-i \hbar \nabla-\frac{e^{*}}{c} \mathbf{A}\right) \psi_{n}\right|^{2} \\
\left.+\frac{\hbar^{2}}{2 m_{c} d^{2}}\left|\psi_{n}(\mathbf{r})-\psi_{n+1}(\mathbf{r})\right|^{2}\right]
\end{gathered}
$$

where $d_{0}$ is the layer thickness, $d$ the layer spacing and $\alpha, \beta, m_{a b}$, and $m_{c}$ phenomenological parameters. We denote by $\tau \equiv \hbar^{2} / 2 m_{c} d^{2}=\left(\xi_{c} / d\right)^{2}$ the dimensionless ratio between the coherence length $\xi_{c}$ perpendicular to the layers and the layer spacing. We take $\mathbf{B}=\nabla \times \mathbf{A}$ as constant and uniform.

We use the lowest Landau level (LLL) approximation which is believed to be valid over a large portion of the vortex liquid regime. We expand the order parameter $\psi_{n}(\mathbf{r})$ in terms of the eigenstates of $\left|\left(-i \hbar \nabla-\frac{e^{*}}{c} \mathbf{A}\right) \psi_{n}\right|^{2}$ and keep only the lowest eigenvalue state. In the symmetric gauge, where $\mathbf{A}=\frac{B}{2}(-y, x, 0)$, the LLL wavefunction is given by $\psi_{n}^{\mathrm{LLL}}(\mathbf{r})=\exp \left(-\mu^{2}|z|^{2} / 4\right) \phi_{n}(z)$ where $\mu^{2}=e^{*} B / \hbar c$ and $\phi_{n}(z)$ is an arbitrary analytic function of $z=x+i y$. In the LLL approximation, the free energy becomes

$$
\begin{aligned}
F[\phi, & \left.\phi^{*}\right]=\sum_{n} d_{0} \int d z^{*} d z\left[\alpha_{H} e^{-\mu^{2}|z|^{2} / 2}\left|\phi_{n}(z)\right|^{2}\right. \\
& +\frac{\beta}{2} e^{-\mu^{2}|z|^{2}}\left|\phi_{n}(z)\right|^{4} \\
& \left.+\tau e^{-\mu^{2}|z|^{2} / 2}\left|\phi_{n}(z)-\phi_{n+1}(z)\right|^{2}\right]
\end{aligned}
$$

where $\alpha_{H} \equiv \alpha+e^{*} B \hbar / 2 c m_{a b}$ changes sign crossing the upper critical field line $H_{c 2}(T)$. Physical properties of the vortex system in the layered superconductor are then determined from the partition function $Z=$ $\int \prod_{n} \mathcal{D} \phi_{n} \mathcal{D} \phi_{n}^{*} \exp \left(-F\left[\phi, \phi^{*}\right] / k_{B} T\right)$.

For a two-dimensional system, that is for a single layer, the temperature and field dependence are all contained within the single dimensionless parameter $\alpha_{2 T} \equiv\left(2 \pi d_{0} / \beta \mu^{2} k_{B} T\right)^{1 / 2} \alpha_{H}$. The inter-layer coupling strength is described by the dimensionless parameter $\tau_{T} \equiv\left(2 \pi d_{0} / \beta \mu^{2} k_{B} T\right)^{1 / 2} \tau$. For the layered system it is very convenient to use the dimensionless field-temperature parameter $\alpha_{T}$ employed in studies of the continuous anisotropic three dimensional GL model: $\alpha_{T}=\left(8 \pi \hbar c / \beta^{\prime} e^{*} B k_{B} T\right)^{2 / 3}\left(\hbar^{2} / 2 m_{c}\right)^{1 / 3} \alpha_{H}$ where $\beta^{\prime}=\left(d / d_{0}\right) \beta$ is the coefficient of the quartic term for the three dimensional GL model. The two-dimensional limit corresponds to $\tau_{T} \rightarrow 0$, while in the limit $\tau_{T} \rightarrow \infty$ for constant $\alpha_{T}$, the system behaves as a continuous three dimensional model. Note that $\alpha_{T}=2^{4 / 3} \tau_{T}^{1 / 3} \alpha_{2 T}$. In terms of $t \equiv T / T_{c 0}$ and $h \equiv H / H_{c 2}(0)$,

$$
\alpha_{T} \sim \frac{1-t-h}{(t h)^{2 / 3}}, \quad \tau_{T} \sim \frac{1}{(t h)^{1 / 2}},
$$


where $T_{c 0}$ is the zero-field transition temperature and $H_{c 2}(0)$ is the straight line extrapolation of the $H_{c 2}(T)$ line near $T_{c 0}$ to zero temperature.

We shall calculate various correlation functions using the parquet approximation. This is a nonperturbative analytic approximation and requires no boundary conditions to be imposed in the direction perpendicular to the magnetic field within each layer. Our system consists of a stack of $N$ layers, on which we impose the periodic boundary condition in the direction along the field $\phi_{n+N}(z)=\phi_{n}(z)$. We introduce the Fourier transform $\widetilde{\phi}_{q}$ of $\phi_{n}$ via

$$
\phi_{n}(z)=\frac{1}{d N} \sum_{q \in 1 \text { st.B.Z. }} e^{i q n d} \widetilde{\phi}_{q}(z),
$$

where $q=2 \pi j / N d$ and we use $N$ integer values of $j$ in the sum such that the wavevector $q \in[-\pi / d, \pi / d)$ belongs to the first Brillouin zone.

One can develop the standard perturbation theory from the given partition function. The bare propagators are given by

$$
\left\langle\widetilde{\phi}_{q^{\prime}}^{*}\left(z^{\prime *}\right) \widetilde{\phi}_{q}(z)\right\rangle_{0}=d^{2} N \delta_{q, q^{\prime}}\left(\mu^{2} / 2 \pi\right) e^{\mu^{2} z^{\prime *} z / 2} G_{0}(q),
$$

where

$$
G_{0}(q)=\left(\frac{k_{B} T}{d_{0}}\right) \frac{1}{\alpha_{H}+2 \tau(1-\cos (q d))} .
$$

Since the magnetic length $\mu^{-1}$ is the only length scale perpendicular to the field direction which appears in the propagator [10], the fully renormalized propagator can also be written as (5) with the renormalized $G(q)$ replacing the bare function $G_{0}(q)$. The renormalized $G(q)$ is determined self-consistently in the parquet approximation.

The main quantity one studies in the parquet approximation is the renormalized connected four-point function, $\left\langle\widetilde{\phi}_{q_{1}}^{*}\left(z_{1}^{*}\right) \widetilde{\phi}_{q_{2}}^{*}\left(z_{2}^{*}\right) \widetilde{\phi}_{q_{3}}\left(z_{3}\right) \widetilde{\phi}_{q_{4}}\left(z_{4}\right)\right\rangle_{c}$ which can be written in terms of the renormalized vertex function $\Gamma\left(q_{1}, q_{2}, q_{3} ; \mathbf{k}\right)=$ $\Gamma\left(q_{1}, q_{2}, q_{3}, q_{1}+q_{2}-q_{3} ; \mathbf{k}\right)$ [10]. Here the wavevector $\mathbf{k}$ lies in the two dimensional space perpendicular to the magnetic field and $q_{i}$ is a wavevector along the field direction. Note that to the lowest order $\Gamma\left(q_{1}, q_{2}, q_{3} ; \mathbf{k}\right)=\Gamma_{B}(\mathbf{k})$ independent of the wavevectors along the field direction.

In order to make a resummation over all parquet diagrams, we note that the contributions to $\Gamma$ can be decomposed into a totally irreducible part and a reducible part which in turn can be written as the sum of three parts $\Pi_{i}(i=$ $1,2,3)$ representing the contributions from three different channels. (A detailed discussion on the diagrammatic decomposition can be found in Ref. [10]). The parquet approximation we employ here corresponds to neglecting in the totally irreducible vertex all the higher order $\left(\mathrm{O}\left(\beta^{4}\right)\right)$ diagrams except the bare vertex function $\Gamma_{B}(\mathbf{k})$ so

$$
\Gamma\left(q_{1}, q_{2}, q_{3} ; \mathbf{k}\right)=\Gamma_{B}(\mathbf{k})+\sum_{i=1}^{3} \Pi_{i}\left(q_{1}, q_{2}, q_{3} ; \mathbf{k}\right) .
$$

Each reducible vertex $\Pi_{i}$ is composed of the irreducible vertex $\Lambda_{i}$ where

$$
\Lambda_{i}\left(q_{1}, q_{2}, q_{3} ; \mathbf{k}\right)=\Gamma_{B}(\mathbf{k})+\sum_{j \neq i} \Pi_{j}\left(q_{1}, q_{2}, q_{3} ; \mathbf{k}\right)
$$

and the renormalized $\Gamma$ via the following Bethe-Salpeter equations:

$$
\begin{aligned}
& \Pi_{1}\left(q_{1}, q_{2}, q_{3} ; \mathbf{k}\right)=-\frac{1}{N} \sum_{p} \widehat{G}(p) \widehat{G}\left(q_{1}+q_{2}-p\right)\left[\Lambda_{1}\left(q_{1}, q_{2}, p\right) \circ \Gamma\left(p, q_{1}+q_{2}-p, q_{3}\right)\right](\mathbf{k}), \\
& \Pi_{2}\left(q_{1}, q_{2}, q_{3} ; \mathbf{k}\right)=-\frac{2}{N} \sum_{p} \widehat{G}(p) \widehat{G}\left(p-q_{1}+q_{3}\right) \Lambda_{2}\left(q_{1}, p-q_{1}+q_{3}, q_{3} ; \mathbf{k}\right) \Gamma\left(p, q_{2}, p+q_{3}-q_{1} ; \mathbf{k}\right), \\
& \Pi_{3}\left(q_{1}, q_{2}, q_{3} ; \mathbf{k}\right)=-\frac{2}{N} \sum_{p} \widehat{G}(p) \widehat{G}\left(p+q_{2}-q_{3}\right)\left[\Lambda_{3}\left(q_{1}, p+q_{2}-q_{3}, p\right) * \Gamma\left(p, q_{2}, q_{3}\right)\right](\mathbf{k}),
\end{aligned}
$$

where the operation o between two arbitrary functions $f(\mathbf{k})$ and $g(\mathbf{k})$ is defined by

$$
\begin{aligned}
(f \circ g)(\mathbf{k})= & \frac{2 \pi}{\mu^{2}} \int \frac{d^{2} \mathbf{k}^{\prime}}{(2 \pi)^{2}} f\left(\mathbf{k}-\mathbf{k}^{\prime}\right) g\left(\mathbf{k}^{\prime}\right) \\
& \times \cos \left(\left(k_{x} k_{y}^{\prime}-k_{y} k_{x}^{\prime}\right) / \mu^{2}\right),
\end{aligned}
$$


and $f * g$ is just the convolution which is the same as the above expression without the cosine term. In (8) we have used the dimensionless form of the propagator function $\widehat{G}(q) \equiv\left(d_{0} \beta \mu^{2} / 2 \pi k_{B} T\right)^{1 / 2} G(q)$. The above equations, (6)), (7) and (8) form a closed set for $\Gamma$ when the renormalized propagator $\widehat{G}(q)$ is known. In the parquet approximation $\widehat{G}(q)$ is determined self-consistently by the Dyson equation:

$$
\begin{aligned}
\widehat{G}^{-1}(q)-\widehat{G}_{0}^{-1}(q) & =\frac{2}{N} \sum_{q^{\prime}} \widehat{G}\left(q^{\prime}\right) \\
& -\frac{2}{N^{2}} \sum_{q^{\prime}, q^{\prime \prime}} \widehat{G}\left(q^{\prime}\right) \widehat{G}\left(q^{\prime \prime}\right) \widehat{G}\left(q+q^{\prime}-q^{\prime \prime}\right) \frac{2 \pi}{\mu^{2}} \int \frac{d^{2} \mathbf{k}}{(2 \pi)^{2}} \Gamma\left(q, q^{\prime}, q^{\prime \prime} ; \mathbf{k}\right) e^{-\mathbf{k}^{2} / 2 \mu^{2}} .
\end{aligned}
$$

Using the solutions to the above equations one can calculate several interesting physical quantities. The structure factor, which is the measure of correlation between vortices in the vortex liquid, is calculated from

$$
\begin{aligned}
\chi_{n-n^{\prime}}\left(\mathbf{r}-\mathbf{r}^{\prime}\right)= & \left\langle\left|\Psi_{n}(\mathbf{r})\right|^{2}\left|\Psi_{n^{\prime}}\left(\mathbf{r}^{\prime}\right)\right|^{2}\right\rangle \\
& -\left\langle\left|\Psi_{n}(\mathbf{r})\right|^{2}\right\rangle\left\langle\left.\Psi_{n^{\prime}}\left(\mathbf{r}^{\prime}\right)\right|^{2}\right\rangle .
\end{aligned}
$$

The structure factor $\Delta_{m}(\mathbf{k})$ used in this paper is then defined by

$$
\Delta_{m}(\mathbf{k}) \equiv\left(\frac{d_{0} \beta}{k_{B} T}\right) e^{\mathbf{k}^{2} / 2 \mu^{2}} \int d^{2} \mathbf{R} e^{i \mathbf{k} \cdot \mathbf{R}} \chi_{m}(\mathbf{R})
$$

Using (9), one can write the generalized Abrikosov ratio $\beta_{A}$ as

$$
\beta_{A} \equiv \frac{\left\langle\left|\Psi_{n}(\mathbf{r})\right|^{4}\right\rangle}{\left\langle\left|\Psi_{n}(\mathbf{r})\right|^{2}\right\rangle^{2}}=\frac{1-N^{-1} \sum_{q} \widehat{G}(q) \widehat{G}_{0}^{-1}(q)}{\left[N^{-1} \sum_{q} \widehat{G}(q)\right]^{2}} .
$$

The Josephson coupling parameter $\eta$ measures the degree of independence between adjacent layers and is given by

$$
\eta \equiv \frac{\left\langle\left|\Psi_{n}(\mathbf{r})-\Psi_{n+1}(\mathbf{r})\right|^{2}\right\rangle}{\left\langle\left|\Psi_{n}(\mathbf{r})\right|^{2}\right\rangle}=\frac{N^{-1} \sum_{q} 2(1-\cos (q d)) \widehat{G}(q)}{N^{-1} \sum_{q} \widehat{G}(q)}
$$

We can also put the above equations in more convenient form as follows. If we introduce

$$
\widetilde{\gamma}\left(q_{1}, q_{2}, q_{3} ; \mathbf{k}\right) \equiv\left[\widehat{G}\left(q_{1}\right) \widehat{G}\left(q_{2}\right) \widehat{G}\left(q_{3}\right) \widehat{G}\left(q_{1}+q_{2}-q_{3}\right)\right]^{1 / 2} \Gamma\left(q_{1}, q_{2}, q_{3} ; \mathbf{k}\right) .
$$

and similary $\widetilde{\lambda}_{i}\left(q_{1}, q_{2}, q_{3} ; \mathbf{k}\right)$ and $\widetilde{\pi}_{i}\left(q_{1}, q_{2}, q_{3} ; \mathbf{k}\right)$ from $\Lambda_{i}\left(q_{1}, q_{2}, q_{3} ; \mathbf{k}\right)$ and $\Pi_{i}\left(q_{1}, q_{2}, q_{3} ; \mathbf{k}\right)$ respectively, and denote the Fourier transform of $\widetilde{\gamma}, \widetilde{\pi}$ and $\widetilde{\lambda}$ by $\gamma\left(\left\{n_{i}\right\} ; \mathbf{k}\right), \pi_{i}\left(\left\{n_{i}\right\} ; \mathbf{k}\right)$ and $\lambda_{i}\left(\left\{n_{i}\right\} ; \mathbf{k}\right)$ with the shorthand notation $\left\{n_{i}\right\}=$ $\left(n_{1}, n_{2}, n_{3}, n_{4}\right)$, then (8) is simplified to

$$
\begin{aligned}
& \pi_{1}\left(\left\{n_{i}\right\} ; \mathbf{k}\right)=-\sum_{n^{\prime}, n^{\prime \prime}}\left[\lambda_{1}\left(n_{1}, n_{2}, n^{\prime}, n^{\prime \prime}\right) \circ \gamma\left(n^{\prime}, n^{\prime \prime}, n_{3}, n_{4}\right)\right](\mathbf{k}) \\
& \pi_{2}\left(\left\{n_{i}\right\} ; \mathbf{k}\right)=-2 \sum_{n^{\prime}, n^{\prime \prime}} \lambda_{2}\left(n_{1}, n^{\prime}, n_{3}, n^{\prime \prime} ; \mathbf{k}\right) \gamma\left(n^{\prime \prime}, n_{2}, n^{\prime}, n_{4} ; \mathbf{k}\right) \\
& \pi_{3}\left(\left\{n_{i}\right\} ; \mathbf{k}\right)=-2 \sum_{n^{\prime}, n^{\prime \prime}}\left[\lambda_{3}\left(n_{1}, n^{\prime}, n^{\prime \prime}, n_{4}\right) * \gamma\left(n^{\prime \prime}, n_{2}, n_{3}, n^{\prime}\right)\right](\mathbf{k}) .
\end{aligned}
$$

The remaining parquet equations can easily be derived as follows:

$$
\lambda_{j}\left(\left\{n_{i}\right\} ; \mathbf{k}\right)=\gamma\left(\left\{n_{i}\right\} ; \mathbf{k}\right)-\pi_{j}\left(\left\{n_{i}\right\} ; \mathbf{k}\right)
$$

for $j=1,2,3$ and

$$
\gamma\left(\left\{n_{i}\right\} ; \mathbf{k}\right)=f_{B}\left(\left\{n_{i}\right\}\right) \Gamma_{B}(\mathbf{k})+\sum_{j=1}^{3} \pi_{j}\left(\left\{n_{i}\right\} ; \mathbf{k}\right),
$$


where

$$
\begin{aligned}
f_{B}\left(\left\{n_{i}\right\}\right)= & \sum_{l} \mathcal{G}\left(l-n_{1}\right) \mathcal{G}\left(l-n_{2}\right) \\
& \times \mathcal{G}\left(n_{3}-l\right) \mathcal{G}\left(n_{4}-l\right)
\end{aligned}
$$

with $\mathcal{G}(n)$ being the Fourier transform of $\widehat{G}^{1 / 2}(q): \mathcal{G}(n)=(1 / N) \sum_{q} \exp (i q n d) \widehat{G}^{1 / 2}(q)$.

\section{RESULTS}

We solved (numerically!) the parquet equations, (14)-(16) for $\gamma\left(\left\{n_{i}\right\} ; \mathbf{k}\right)$ with Eq. (9) for the propagator $G(q)$. One could use equivalently the set of equations, (6)-(8) for $\Gamma\left(\left\{q_{i}\right\} ; \mathbf{k}\right)$. It is just a matter of convenience. In the present analysis the former was used. In solving we start from some initial functions, $\lambda_{i}, \gamma$ and $G(q)$ and update these functions iteratively using (14)-(16) and (9). As mentioned above, the main numerical difficulty compared to the two-dimensional case is that $\gamma$ and $\lambda_{i}$ now involve three extra indices in addition to the two-dimensional momentum $\mathbf{k}$. A large amount of computer memory and CPU time is required as the number of layers becomes large. In this paper we only consider a small system where the number of layers is four and try to see what effect the coupling between the layers has on the two-dimensional parquet results. At high temperatures we find that the iteration converges very quickly, but as the temperature is lowered the convergence gets slower, and furthermore we have to use the results obtained at a nearby temperature as the initial values for $\lambda_{i}$ and $\gamma$ in order to get rapid convergence.

From the solutions $\Gamma$ and $G(q)$ to the parquet equations for given $\alpha_{2 T}$ and $\tau_{T}$, we calculated the thermodynamic quantities introduced in the previous section. The three-dimensional temperature parameter $\alpha_{T}$ can be obtained from the two parameters. Figures 1 and 2 show the Abrikosov ratio $\beta_{A}$, defined in Eq. (11) and the Josephson coupling parameter $\eta$ in Eq. (12) as functions of $\alpha_{T}$ for various values of the interlayer coupling $\tau_{T}$. We find that for some values of $\tau_{T}$, these thermodynamic quantities show abrupt changes as $\alpha_{T}$ is varied. But this behavior disappears for $\tau_{T} \geq 0.11$ when now $\beta_{A}$ and $\eta$ just decrease smoothly without showing any sudden drops as the temperature is lowered. These features can be explained by the existence of a first-order transition in the vortex liquid and of a critical end-point: the first-order nature of the transition gets weaker as the inter-layer coupling strength $\tau_{T}$ increases, eventually disappearing at the critical end-point at $\tau_{T} \simeq 0.11$. 


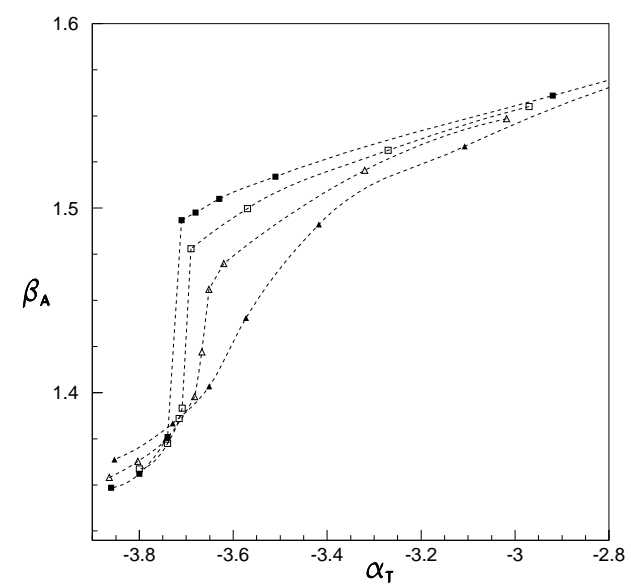

FIG. 1. The Abrikosov ratio $\beta_{A}$ as a function of temperature parameter $\alpha_{T}$ for different inter-layer coupling strengths $\tau_{T}=0.1$ (filled squares), 0.105 (open squares), 0.11 (open triangles) and 0.12 (filled triangles). The dotted lines are guides to the eye.

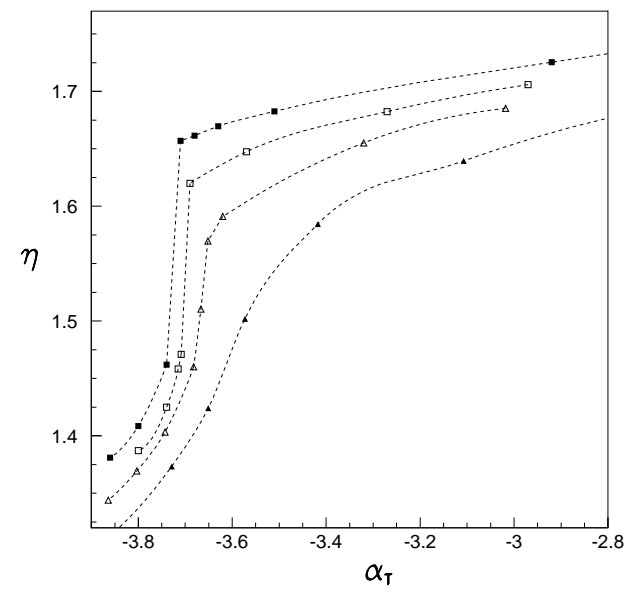

FIG. 2. The Josephson coupling parameter $\eta$ as a function of temperature parameter $\alpha_{T}$ for different inter-layer coupling strengths $\tau$. The symbols are the same as in Fig. 1. The dotted lines are guides to the eye.

The sudden changes in these quantities occur in a very narrow range of the temperature parameter $\alpha_{T}$. We believe it is not a crossover which just happens to resemble a first order transition, since we find instabilities in obtaining the solutions to the parquet equations within this narrow range of $\alpha_{T}$. This is illustrated in Fig. 3. It shows a typical example of how thermodynamic quantities such as $\beta_{A}$ or $\eta$ evolve as we iteratively solve the parquet equations in the vicinity of the transition. Well above the transition, $\beta_{A}$ converges rather quickly as can be seen in Fig. 3 . This is also the case well below the transition. However, as the transition is approached from above the convergence gets slower, then we reach a very narrow region of $\alpha_{T}$ where the iteration appears not to be converging. We find this kind of instability for the cases where $\tau_{T}<0.11$, while no instability is seen for $\tau_{T} \geq 0.11$. This is in fact how we locate the critical end-point in our model. Since the iteration method works well only if we use initial functions which are close to the actual solutions, we conclude that this instability signals a sharp change emerging in the system. Strictly speaking, there should exist a solution for any value of $\alpha_{T}$ even if there is a first order transition. Therefore the instability does not imply that there is no convergent solution. It just tells us that it is very hard to get a solution using the iteration method employed here. (It is interesting to note that the solution below the transition was in 
fact found after a very long iteration). For this reason, although we find that the size of the first-order transition-like jumps in thermodynamic quantities such as $\beta_{A}$ decreases as the critical end-point is approached, it is quite hard to determine accurately the size of these jumps.

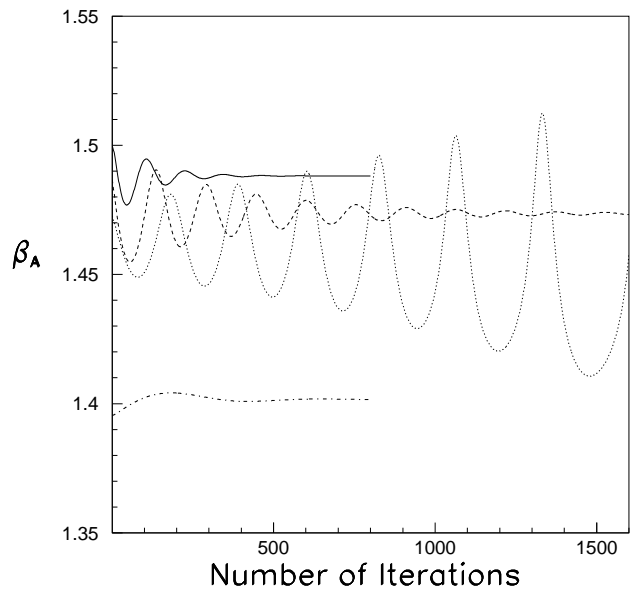

FIG. 3. The evolution of the Abrikosov ratio $\beta_{A}$ in the iteration of the parquet equations. The inter-layer coupling is fixed at $\tau_{T}=1.075$. The three dimensional temperature parameter $\alpha_{T}$ is given by -3.594 (solid), -3.654 (dashed), -3.684 (dotted), and -3.690 (dot dashed).

The first order transitions we obtain here are between two vortex liquid phases with the phase below the transition being more correlated. This can be seen in Fig. 4 where the structure factor $\Delta_{n}(K)$ defined in Eq. (10) is shown at temperatures above and below the transition. In both cases, there is no crystalline long-range order present in the system. We note that the first peak at $K \simeq 2.69$, which is the value of the first reciprocal lattice vector of a triangular lattice, represents the crystalline order developing in the plane perpendicular to the magnetic field. Comparing the structure factors above and below the transition, the difference is most significant for the case of $n=2$. This tells us that the state below the transition is a vortex liquid with larger correlation along the field direction. Therefore the transition we find is more like a decoupling transition occuring in the vortex liquid. This can also be seen in the drop in $\eta$ (Fig. 2) as the temperature is lowered, since $\eta$ is largest when neighboring layers are more or less independent.

(a)

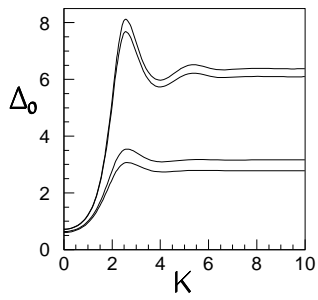

(b)

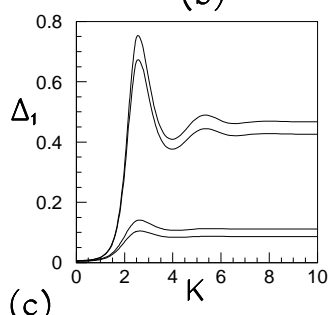

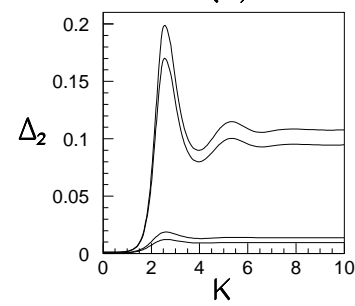

FIG. 4. The structure factor $\Delta_{n}(K)$ at $\tau_{T}=0.105$ and four different values of $\alpha_{T}$. The dimensionless two-dimensional wavevector $K \equiv k / \mu$. (a) $\mathrm{n}=0$,(b) $\mathrm{n}=1$, and (c) $\mathrm{n}=2$. For all cases, $\alpha_{T}=-3.715,-3.709,-3.685$ and -3.566 reading from top to bottom. 
Figure 5 shows a collection of the first-order transition points in the $\alpha_{T}-\tau_{T}$ plane and the location of the critical end-point. The transition line is almost independent of $\alpha_{T}$. Although we were only able to study the region close to the critical end-point, the nearly $\alpha_{T}$-independent transition line is consistent with the result obtained in Ref. [8]. We found it very hard to extend this line to the small- $\tau_{T}$ region. Since the size of the jump gets bigger as we move away from the critical end-point to the small- $\tau_{T}$ region, a solution below the transition is going to be very different from the one above, which makes the parquet equations hard to solve by iteration. According to Eq. (3) the transition line which ends at large $\tau_{T}$ corresponds to a critical end-point at low magnetic field. In the H-T space, the shape of the phase boundary looks like the one found in Ref. [8], which is consistent with the experimental results on YBCO-type superconductors. However, we do not expect that the actual position of the transition line in our work can be directly compared with experiment since numerical difficulties have limited us to studying only a small number of layers. The transition temperatures $\left(\alpha_{T} \sim-3.7\right)$ in our model are higher than those found in Ref. [8] $\left(\alpha_{T} \sim-7.0\right)$, and therefore are also higher than the experimental values.

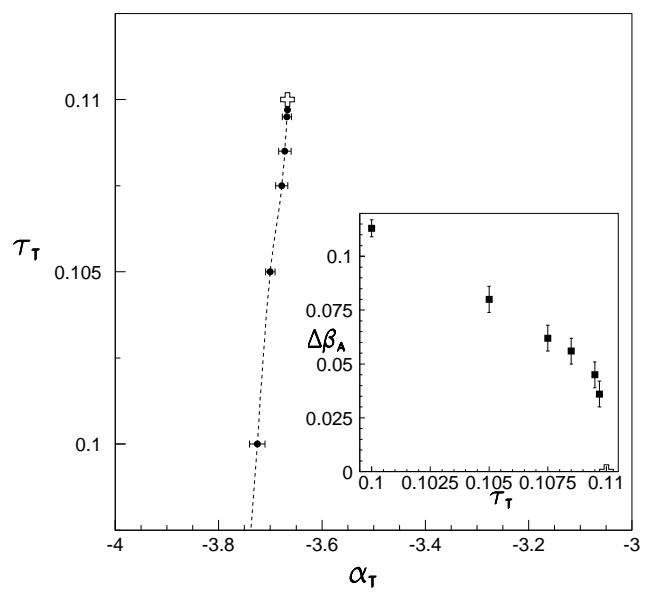

FIG. 5. The first order transition line and the location of the critical end-point (cross) in the $\alpha_{T}-\tau_{T}$ space. The inset shows how the size of the jump in $\beta_{A}$ at the transition decreases as the critical end-point is approached.

To summarize we have applied a nonperturbative analytic method to the vortex liquid system in a layered superconductor. The inter-layer coupling produces a first-order transition line which ends at a critical end-point at low fields, whereas for a purely two-dimensional system there are no transitions of any kind within the parquet approximation. The results are consistent with the first-order transition being a decoupling transition between two vortex liquid phases. Clearly in order to extend this method to other situations eg. lower temperatures, the effects of disorder and above all, more layers, we have to devise a way to speed up the convergence of the iterative solution to the parquet equations. One possible method might be to use a combination of solutions obtained in previous steps as the next stage of the iteration [1].

\section{ACKNOWLEDGMENTS}

J. Y. was supported by the Korea Research Foundation Grant(KRF-1999-015-DI0070).

[1] G. Blatter et al., Rev. Mod. Phys. 66, 1125 (1994).

[2] W. K. Kwok, J. Fendrich, S. Fleshler, U. Welp, J. Downey, and G. W. Crabtree, Phys. Rev. Lett. 72, 1092 (1994).

[3] R. Liang, D. A. Bonn, and W. N. Hardy, Phys. Rev. Lett. 76, 853 (1996). 
[4] A. Schilling, R. A. Fisher, N. E. Philips, U. Welp, D. Dasgupta, W. K. Kwok, and G. W. Crabtree, Nature (London) 382, 791 (1996).

[5] A. Junod, M. Roulin, J.-Y. Genoud, B. Revaz, A. Erb, and E. Walker, Physica C 275, 245 (1997).

[6] M. Roulin, A. Junod, A. Erb, and E. Walker, Phys. Rev. Lett. 80, 1722 (1998).

[7] A. Schilling, R. A. Fisher, N. E. Philips, U. Welp, W. K. Kwok, and G. W. Crabtree, Phys. Rev. Lett. 78, 4833 (1997).

[8] A. K. Kienappel and M. A. Moore, Phys. Rev. B 60, 6795 (1999).

[9] J. Hu and A. H. MacDonald, Phys. Rev. B 56, 2788 (1997); R. Sás̆ik and D. Stroud, Phys. Rev. Lett. 75, 2582 (1995).

[10] J. Yeo and M. A. Moore, Phys. Rev. Lett. 76, 1142 (1996); Phys. Rev. B 54, 4218 (1996).

[11] K.-C Ng, J. Chem. Phys. 61, 2680 (1974). 